\title{
Guest editorial to the special section on MODELS 2012
}

\author{
Jürgen Kazmeier • Perdita Stevens
}

Published online: 4 June 2014

(C) Springer-Verlag Berlin Heidelberg 2014

\section{Introduction}

In this special section, we are happy to present three papers that resulted from invitations we made to the authors of some of the best papers presented at MODELS 2012. MODELS 2012 was the ACM/IEEE 15th International Conference on Model Driven Engineering Languages and Systems and took place in Innsbruck, Austria, from September 30 to October 5, 2012.

The conference has established itself as one of the key international venues for the presentation of scientific results in the domain of model-driven engineering and related topics such as software modeling and model transformation. A total of 50 papers were selected for publication in the conference proceedings from 258 papers submitted for review from over 30 different countries. As usual, both technical and experience papers were accepted, and separate program subcommittees evaluated the two kinds of submissions. There were three keynote speakers: Janos Sztipanovits (Vanderbilt/USA), who spoke about Model Integration Languages; Thomas Henzinger (IST/Austria) who spoke about Quantitative Reactive Modelling; and from industry, Stephan Thesing (EUROCOPTER/Germany), whose presentation concerned ideas and requirements for model-driven software and system development. The speakers kindly provided invited papers for the conference proceedings.

The selection of papers to invite was made in consultation with Robert France, who chaired the Foundations Track of the conference, but did not wish to edit this special issue in

\section{J. Kazmeier}

Siemens, Berlin, Germany

P. Stevens $(\varangle)$

University of Edinburgh, Edinburgh, Scotland

e-mail: Perdita.Stevens@ed.ac.uk view of his role as Editor in Chief. This is not mere republication: papers had to be significantly extended and improved, compared with the conference papers, to be accepted, and the new versions underwent a full journal review process. The papers here are those which could quickly be seen to be of high enough quality; several other papers were considered promising, but not ready for publication in their present form. They are undergoing revisions, and we hope they may be published in future issues of SoSyM.

\section{Selected papers}

First, from the Foundations track, we have Example-driven meta-model development by Jesús J. López-Fernández, Jesús Sanchez Cuadrado, Esther Guerra and Juan de Lara. This paper addresses an important practical problem for the adoption of model-driven development: how are the required metamodels to be developed, given that the people with the domain expertise to do it may not be metamodeling experts? Here the authors present an interactive and iterative approach that involves deducing the metamodel from model fragments.

Next, from the Applications track, we have Evaluating the Effort of Composing Design Models: A Controlled Experiment by Kleinner Farias, Alessandro Garcia, Jon Whittle, Christina Chavez and Carlos Lucena. This paper compares specification-based and heuristic-based composing techniques, e.g., evolving UML diagrams, and is closing a gap by quantitative analysis of the effects on developers' effort detecting and resolving inconsistencies in the output composed models. Is the use of manual heuristics for model composition outperforming their automated counterparts? This is an important contribution to support modeling in practice. 
Finally, also from the Applications track, we have Applying UML/MARTE on Industrial Projects: Challenges, Experiences and Guidelines by Muhammad Zohaib Iqbal, Shaukat Ali, Tao Yue and Lionel Briand. In this paper, the authors report their experience on applying the UML profile MARTE (Modeling of Real-Time and Embedded Systems) to three distinct industrial problems. The challenges and lessons learned out of these case studies are of great value for practitioners. They are presented here as a convincing framework of detailed guidelines, which gives interesting insights on real-world industrial application needs.

Acknowledgments We would like to thank the Editors-In-Chief of SoSyM, Bernhard Rumpe and Robert France, for welcoming this special issue. We thank the referees, both for the conference and for the special issue, whose comments have, we believe, helped to ensure that these papers will be interesting and useful reading. Martin Schindler and the SoSyM editorial office have been helpful throughout, and we are most grateful for their support. Last but not least, we thank the authors for submitting their work and for taking notice of the referees' comments.

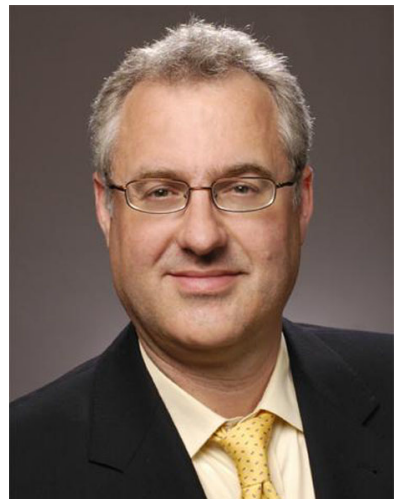

Jürgen Kazmeier holds a major degree in Mathematics and a Ph.D. in Computer Science from the Technical University of Munich. $\mathrm{He}$ has worked on software development processes, methods, and tools, especially on Modeling Languages, Requirements Engineering and Global Software Development. He hold management positions at Siemens in USA, Austria and Germany in different areas, e.g., Research, R\&D, Consulting, IT-Services. Currently, he is Vice President of Enterprise Services, Siemens Healthcare in Erlangen, Germany.

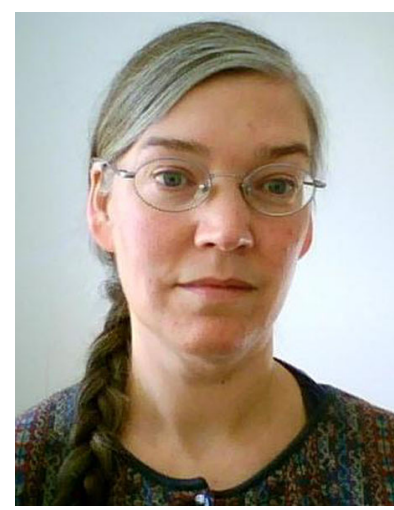

Perdita Stevens is a Professor of Mathematics of Software Engineering in the School of Informatics at the University of Edinburgh. She has been interested in model-driven development since before UML; she chaired the 2003 edition of the precursor of the MODELS conference, then called UML. Currently, her principal interests are in mathematics of software engineering, especially bidirectional transformations. She chairs the Bx steering committee, and serves on the editorial boards of both SoSyM and Theoretical Computer Science. 